\title{
ANGULAR MOMENTUM IN ELLIPTICAL GALAXIES
}

\author{
Rosemary F.G. Wyse ${ }^{1,2}$ and Susana Lizano ${ }^{2}$ \\ 1. Space Telescope Science Institute, Baltimore, MD 21218. \\ 2. Astronomy Department, University of California, Berkeley, CA 94720
}

\begin{abstract}
We have used the available published observations of the rotational properties of elliptical galaxies to test theories of galaxy formation which predict an anti-correlation between the angular momentum of a galaxy and its initial overdensity and hence formation epoch. We find that the prediction, at least in its simplest form, is not supported by the data for well studied elliptical galaxies which have a range in rotational support.
\end{abstract}

\section{DISCUSSION}

Major requirements of a theory of galaxy formation are that it offer an explanation of the differences between elliptical and spiral galaxies and an understanding of the correlations among dynamical parameters within a Hubble type. The crucial parameter determining the final morphological type of a proto-galactic density perturbation has recently been proposed to be initial overdensity, relative to the mean overdensity on a galactic scale ${ }^{1,2}$. This proposal, which works best if the universe is dominated by cold dark matter ${ }^{1}$, predicts that the nonrotating (elliptical) galaxies form from those density perturbations on a galaxy scale which are very overdense compared to the average - out on the Gaussian tail - and hence collapse at larger redshift than the rms (one-sigma) galaxy-sized perturbation. Initial overdensity is anti-correlated with initial angular momentum, along the Hubble sequence and within a Hubble type, which is the prediction we investigate here.

Davies et al have published measurements of the rotation parameter $(\mathrm{v} / \sigma)$ for a sample of elliptical galaxies, and find a large range of rotational support within this Hubble type. We have attempted to derive initial pre-collapse values for the galaxy densities, assuming that the stellar $M / L=8$, there are dark halos of ten times the stellar masses, and the galaxies underwent dissipationless collapse by a factor 2 in radius. The velocity dispersion is assumed constant, while the rotation velocity increased as expected for non-selfgravitating collapse. An anti-correlation between initial $\mathrm{v} / \sigma$ and density is not compatible with these data, although the possibilities of variable collapse factor and dissipation, together with the spread in masses for the sample are complications. Thus in Figure 1a we show density versus temperature (or velocity dispersion) for these ellipticals. The different symbols reflect different levels of rotational support. The rms 1-sigma relationships for clustering hierarchies of initial power spectrum slope $n=-1,-2$ are as indicated (cold dark matter being somewhere between the two). If rotation were determined mainly by iritial everdensity in the way suggested, the low-sigma rapidly rotating 
ellipticals and the high-sigma slowly rotating ellipticals should be offset from each other, perpendicular to the rms line ${ }^{1}$. This does not appear to be the case. If one were to argue for a variable collapse factor, then one could produce an offset by requiring ad hoc that the most highly rotating galaxies collapsed by a factor three more than the slowly rotating galaxies. The likelihood that elliptical galaxies underwent dissipation while forming will cause a spread in the densities, so use of a conserved quantity like mass may be preferred (assuming negligible secondary infall). Mass against temperature is plotted in Figure $1 b$, the hierarchy lines and symbols as before. Again we see that galaxies of different levels of rotational support are well-mixed. Subsets could be moved relative to each other by adopting a different $M / L$, but an offset could be achieved only if the slowly rotating ellipticals had an $\mathrm{M} / \mathrm{L} \gtrsim 5$ times that of the more rotationally supported ellipticals.
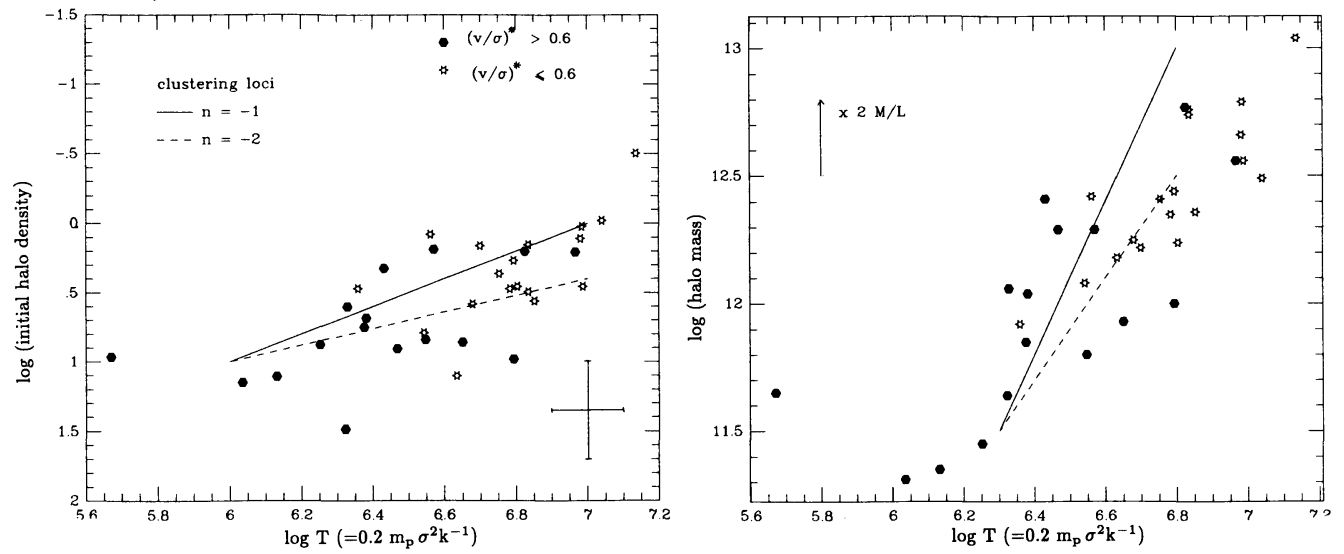

Figure 1. (a) Initial density as a function of temperature, defined as labelled. (b) Total mass against temperature.

\section{CONCLUSIONS}

It appears that the simplest theories which predict an anti-correlation between angular momentum and initial density are not strongly supported by the data, at least for well studied elliptical galaxies. As indicated above, theory and data may be reconciled if one introduces a variable amount of dissipation among ellipticals, leading to different collapse factors before star formation. This may be possible if those perturbations which form the slowly rotating galaxies collapsed in the Compton cooling era ${ }^{4}$, while those which rotate did not. However, since it is extremely difficult to follow how the angular momentum of a galaxy depends on initial conditions, due to problems of identifying which material will eventually be incorporated, it may be that a more complicated scenario of Gaussian perturbations suffices.

\section{REFERENCES}

1. Blumenthal, G.R., Faber, S.M., Primack, J.R., \& Rees, M.J., 1985. Nature, 311, 517.

2. Bardeen, J.M., Bond, J.R., Kaiser, N., \& Szalay, A.S., 1986. Ap. J., 304, 15.

3. Davies, R.L., Efstathiou, G., Fall, S.M., Illingworth, G.D., \& Schechter, P.L., 1983. Ap. J., 266, 41.

4. Lizano, S., \& Wyse, R.F.G., 1986. In preparation. 